\title{
New insights into real-time multiprotein fluorescence microscopy
}

\author{
Cris G. dos Remedios ${ }^{1}$
}

Received: 5 March 2017 / Accepted: 5 March 2017 /Published online: 21 March 2017

(C) International Union for Pure and Applied Biophysics (IUPAB) and Springer-Verlag Berlin Heidelberg 2017

There are several reasons why I think the review by Bunt and Wouters (2017) in this issue of Biophysical Reviews deserves to be singled out. My choice does reflect a long-standing personal research interest in fluorescence resonance energy transfer (FRET) spectroscopy (dos Remedios et al. 1972; dos Remedios and Moens 1995), but before I discuss the Bunt and Wouters review, a little background may be useful.

Fluorescence is a key tool in biophysics. However, interpreting data from fluorescence spectroscopy and fluorescence microscopy is complex. When it is used quantitatively, interpretations of FRET rely on the seminal work of its inventor, Theodor Förster (1946). Simply put, he defined the relationship between a single fluorescent donor probe and a single acceptor probe in solution, thereby making it possible to quantify the distance between the probes.

When an excited fluorescent donor probe comes close (10$100 \AA$ ) to a fluorescent acceptor probe, a radiationless transfer of energy occurs from the donor to the acceptor, resulting in a decrease in the donor fluorescence intensity (and lifetime) and an increase in acceptor fluorescence. The Förster equation states that the rate of energy transfer depends on several parameters, most of which are readily measured. Fluorescence intensity depends on the donor quantum yield (brightness), the refractive index of the medium, the absorption spectrum of the acceptor, and a constant (Avogadro's number). However, it also depends on the Förster orientation factor $\left(\mathrm{K}^{2}\right)$. This parameter is indeterminant and is generally assigned the value of $2 / 3$ on the assumption that the donor and acceptor probes can

Cris G. dos Remedios

crisdos@anatomy.usyd.edu.au

1 Bosch Institute, The University of Sydney, Anderson Stuart Building F13, Sydney 2006, Australia freely precess during the lifetime of the donor. dos Remedios and Moens (1995) argued that this assumption was valid in practice, and that, even if the absolute value was slightly off the mark, the measurement of relative differences in FRET efficiency based on an assumed constant value of $\kappa^{2}$ could provide an excellent measure of conformational change in the donor/acceptor framework architecture interpreted in terms of average separation distance.

FRET experiments are usually performed in vitro by covalently attaching extrinsic donor and acceptor probes to different proteins, enabling us to monitor protein-protein interaction. Extrinsic probes like the Alexa series are small (molecular weight $389 \mathrm{Da}$ ) compared to proteins and are widely used because they resist photobleaching. However, donor probes need not necessarily be extrinsic. Tryptophan (dos Remedios et al. 1972) and, to a lesser extent, tyrosine (Antosiewicz and Shugar 2016) are good examples.

Perhaps the ultimate intrinsic probe is the fluorescent $\beta$ barrel scaffold core of the naturally occurring green fluorescent protein (GFP). It can now be expressed by genetic engineering of GFP-based genes into any protein gene of interest to create a chimeric protein. The fluorescent $\beta$-barrel scaffold structure can be modified in different ways (Germond et al. 2016) to make variants such as yellow fluorescent protein (YFP), blue fluorescent protein (BFP), and red fluorescent protein (RFP). Furthermore, fusion proteins have now been created that can measure intracellular changes in $\mathrm{pH}$ and chloride and calcium ion concentrations. Other variants can sense redox state, membrane potential, and changes in temperature and pressure in living cells. The best thing about them is that they can be incorporated into any protein gene and expressed in living cells. However, these GFP derivatives are much larger $(\sim 30 \mathrm{kDa})$ than standard fluorescent probes, and this raises the question of whether this bulk $(\sim 3 \mathrm{~nm})$ impacts on their ability to precess sufficiently freely to satisfy $\kappa^{2}$ ? 
Now, let me come to the Bunt and Wouters review. They question whether the $\kappa^{2}$ factor $=2 / 3$ always applies, and they discuss two examples where multiple GFP variant probes are co-expressed in living cells.

Antenna effect They point out that the analysis of FRET can get more complex when multiple acceptors are used when performing fluorescence microscopy in living cells. They note that the probability of FRET increases with the number of acceptor probes and this "antenna effect" (where a signal can be detected over greater distances) is more obvious at longer FRET distances. Most FRET measurements fall within the range $65-100 \AA$, but with the antenna effect, significantly longer distances can be realized.

Surplus effect The second confounder when using multiple $\beta$-barrel scaffold acceptors is the "surplus" effect. This makes it difficult to accurately quantify FRET. It deals with the case where a donor probe is attached to two different acceptor probes in a ternary complex. Here, the measured FRET efficiency is greater than the simple sum of the FRETs for the donor-acceptor\#1 and donor-acceptor\#2 pairs, enabling longer (surplus) distances to be determined.

The surplus effect is important when measuring FRET distances using multiple fluorescent $\beta$-barrel donors and acceptors using fluorescence microscopy of living cells. Rather than monitoring fluorescence intensity, Bunt and Wouters discuss the use of fluorescence lifetime imaging microscopy (FLIM). They point out that these systems particularly benefit from using normalized transfer rates which can be experimentally measured by acceptor photobleaching microscopy and by FLIM.

They review several reports in which the molecular details of rapid, biologically complex events such as apoptosis and transcription have been followed in real time in live cells with multicolor images that quantify intermolecular distances.

I doubt Förster would have anticipated these developments.

On a completely different subject, I am pleased to report that Biophysical Reviews is growing stronger. It is now publishing significantly more reviews per issue than ever before. The graph below shows that the number of articles per issue has trebled since the journal began in 2009 , and in the two years following 2014, when there was a small reversal of this trend, the article numbers increased by $30 \%$ each year. This year, the journal will publish bi-monthly rather than quarterly, and based on the numbers of articles expected in the next four Special Issues, that increase is likely to continue.

Contributors will be pleased to read that Biophysical Reviews is now listed in PubMed Central and will have all articles indexed in PubMed from this year. Furthermore,
Springer Nature has beta-tested and now adopted a new policy that enables authors to share data and manuscripts more freely. This facility is called "SharedIt" and is reviewed in greater depth in this issue by Damien Hall (Hall 2017).

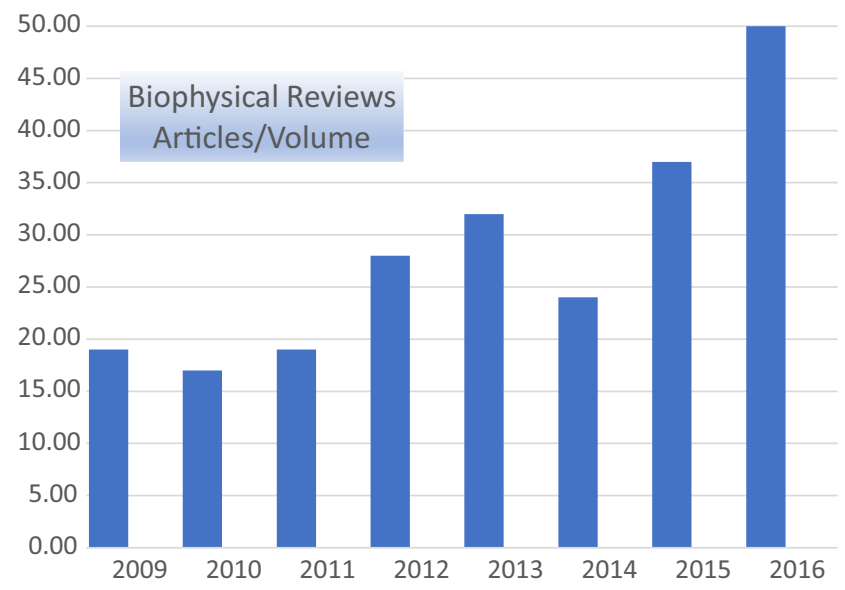

Cris dos Remedios

Editor-in-Chief

\section{Compliance with ethical standards}

Conflict of interest Cris G. dos Remedios declares that he has no conflict of interest.

Ethical approval This article does not contain any studies with human participants or animals performed by the author.

\section{References}

Antosiewicz JM, Shugar D (2016) UV-Vis spectroscopy of tyrosine sidegroups in studies of protein structure. Part 2: selected applications. Biophys Rev 8:163-177. doi:10.1007/s12551-016-0197-7

Bunt G, Wouters FS (2017) FRET from single to multiplexed signaling events. Biophys Rev 9 (in press)

dos Remedios CG, Millikan RGC, Morales MF (1972) Polarization of tryptophan fluorescence from single striated muscle fibers. A molecular probe of contractile state. J Gen Physiol 59:103-120

dos Remedios CG, Moens PDJ (1995) Fluorescence resonance energy transfer spectroscopy is a reliable "ruler" for measuring structural changes in proteins. Dispelling the problem of the unknown orientation factor. J Struct Biol 115:175-185

Förster T (1946) Energiewanderung und Fluoreszenz. Naturwissenschaften 33:166-175

Germond A, Fujita H, Ichimura T, Watanabe TM (2016) Design and development of genetically encoded fluorescent sensors to monitor intracellular chemical and physical parameters. Biophys Rev 8:121138. doi:10.1007/s12551-016-0195-9

Hall D (2017) Innovations in publication: free sharing of all Biophysical Reviews' content. Biophys Rev 9 (in press) 